\title{
Localization of the Centers of Intramuscular Nerve Dense Regions of the Rotator Cuff Muscles: A Guide for Botulinum Toxin A Injection for Spasticity Accompanied by Shoulder Pain
}

\author{
Localización de los Centros de las Regiones Densas del Nervio Intramuscular \\ de los Músculos del Manguito Rotador: Una Guía para la Inyección de \\ Toxina Botulínica A por Espasticidad con Dolor de Hombro
}

Huaixiang Luo; Songling Ji \& Shengbo Yang

LUO, H.; JI, S. \& YANG, S. Localization of the centers of intramuscular nerve dense regions of the rotator cuff muscles: A guide for botulinum toxin a injection for spasticity accompanied by shoulder pain. Int. J. Morphol., 38(2):435-443, 2020.

SUMMARY: To accurately localize the centers of intramuscular nerve dense regions (CINDRs) of rotator cuff muscles. Twenty adult cadavers were used. The curves on skin connecting the superior angle of scapula with the acromion, and with the inferior angle of scapula were designed as the horizontal $(\mathrm{H})$ and longitudinal $(\mathrm{L})$ reference lines, respectively. One side of the rotator cuff muscles were removed and subjected to Sihler's staining to show intramuscular nerve dense regions, and the contralateral muscles' CINDRs were labeled with barium sulfate and scanned by computed tomography (to determine body surface projection points $(\mathrm{P})$ ). The intersection of the longitudinal line from point $\mathrm{P}$ to line $\mathrm{H}$, and that of the horizontal line from point $\mathrm{P}$ to line $\mathrm{L}$, were recorded as $\mathrm{P}_{\mathrm{H}}$ and $\mathrm{P}_{\mathrm{L}}$, respectively. The projection of CINDRs on the anterior body surface across the saggital plane was defined as $\mathrm{P}^{\prime}$ and the line connecting $\mathrm{P}$ to $\mathrm{P}^{\prime}$ was recorded as Line PP'. Percentage positions of CINDRs of $\mathrm{P}_{\mathrm{H}}$ and $\mathrm{P}_{\mathrm{L}}$ on lines $\mathrm{H}$ and $\mathrm{L}$, and the depths on line PP' were determined under the Syngo system. Two, four, one, and one CINDRs were identified in supraspinatus, infraspinatus, teres minor, and subscapularis muscles, respectively. The positions of $\mathrm{P}_{\mathrm{H}}$ of these CINDRs on the $\mathrm{H}$-line are as follows: supraspinatus, $25.43 \%$ and $26.59 \%$; infraspinatus, $53.85 \%, 34.63 \%, 35.96 \%$ and $58.17 \%$; teres minor, $74.50 \%$; and subscapularis, $20.33 \%$. The $\mathrm{P}_{\mathrm{L}}$ on the L-line: supraspinatus, 11.09 $\%$ and $14.83 \%$; infraspinatus, $21.59 \%, 27.93 \%, 48.55 \%$ and $57.52 \%$; teres minor, $68.28 \%$; and subscapularis, $52.82 \%$. The depth on line PP': supraspinatus, $24.83 \%$ and $25.40 \%$; infraspinatus, $21.55 \%, 16.10 \%, 10.01 \%$ and $8.14 \%$; teres minor, $13.27 \%$; and subscapularis, $22.88 \%$. The identification of these CINDRs should provide the optimal target position for injecting botulinum toxin A to treat rotator cuff muscles spasticity accompanied by shoulder pain and to improve the efficiency and efficacy of blocking target localization.

KEY WORDS: Rotator cuff muscles; Spasticity; Intramuscular nerve dense regions; Botulinum toxin A; Blocking target localization.

INTRODUCTION

Rotator cuff muscles spasticity may result from stroke, brain trauma, or spinal cord injury (Maynard et al., 1990; Joynt, 1992; Simpson et al., 2009). Individuals suffering from rotator cuff muscles spasticity usually present with shoulder pain (Van Ouwenaller et al., 1986; Yelnik et al., 2007) and limited shoulder joint movement affecting patient's daily life (Chae et al., 2007). Currently, treatment methods of rotator cuff muscles spasticity include intra-articular injection of drugs, such as triamcinolone (Snels et al., 2000), percutaneous electric stimulation therapy (Renzenbrink \& Ijzerman, 2004), suprascapular nerve block (Wassef, 1992; Shanahan et al., 2003; Picelli et al., 2017), subscapular nerve block (Hecht, 1992) and intramuscular injection of botulinum toxin A (BTX-A ) (Pedreira et al., 2008). BTX-A injection is becoming increasingly popular by blocking the release of acetylcholine from the presynaptic membrane at the motor endplate (Friedman et al., 2000; Kinnett, 2004; Park \& Chung, 2018). However, the visualization of the motor endplate was limited because it can only be stained on fresh specimens. As a result, the motor endplate band of many muscles, including rotator cuff muscles, have not been studied. Recent studies have shown that intramuscular nerve dense regions (INDR) are at the same location of the endplate bands (Amirali et al., 2007; Tang et al., 2018), and INDR may serve as an alternative target for BTX-A blocking injections. 
Regarding the four rotator cuff muscles, the intramuscular nerve distribution of subscapularis were described by a researcher using microdissection and Sihler 's staining methods. This study has provided useful information for BTX-A injection (Warden et al., 2014; Cho et al., 2019) . But the two studies have yet to locate INDR and the center of intramuscular nerve dense regions (CINDR). As for the other three rotator cuff muscles, no reports about their intramuscular nerve distributions have been seen.

Currently, targets of BTX-A blocking injection are located via clinical methods including hand palpation, electromyography, electrical stimulation, and ultrasonic (Childers et al., 1998; Yelnik et al., 2007; Unlu et al., 2008; Rha et al., 2012; Rasool et al., 2017). But these methods are limited to a certain extent such as they still cannot accurately locate the target and avoid the pain caused by exploratory puncture and high-dose injection. Based on the Sihler's staining technique, which can clearly display the overall distribution pattern of intramuscular nerves within a muscle and reveal these fibers to naked eyes, this study intends to accurately localize the CINDRs of all rotator cuff muscles and refer the locations to body surface boney landmarks. We hope to provide accurate blocking targets for BTX-A injection to rotator cuff muscles undergoing spasticity and generating shoulder pain.

\section{MATERIAL AND METHOD}

Specimens and ethics: This study was performed on 20 formalin-fixed adult cadavers (14 men, 6 women) age 3075 at the time of death with no history of neuromuscular diseases and Shoulder-back deformity. The collection and use of specimens were approved by the ethics committee of our school.

Gross anatomy: Cadavers were placed in prone position. Two transverse skin incisions were made at the level of cervical spine 7 and thoracic spine 8 , and a longitudinal skin incision was made between the two transvers incisions along the spinous processes. The skin flaps were turned laterally to the posterior margin of the sternocleidomastoid muscle at the superior end and to the anterior axillary line at the inferior end. Superficial fascia was removed. The deltoid muscle was detached from its proximal attachments and turned downward to expose the three posterior rotator cuff muscles. The subscapularis muscle was exposed with cadaver in supine position and the pectoralis major and pectoralis minor muscles were detached from thoracic wall and turned laterally or superiorly. Observations of muscle shape, nerve innervation and number of neuromuscular branches, and location of nerve entry points were observed on all four rotator cuff muscles (supraspinatus, infraspinatus, subscapularis and teres minor) before completely removing them at their bone attachment sites. The extra fascia on muscle surfaces were removed and the muscles were subjected to Sihler's intramuscular nerve staining.

Sihler's staining method showed intramuscular nerve dense regions: Rotator cuff muscles were removed from the left side of 10 cadaver and the right side of the other 10. Samples were carried through the Sihler's staining process we used before (Tang et al.). The reagent concentration and processing time were adjusted as appropriate. Briefly, specimens were soaked in $3 \%$ hydroxide and $0.2 \%$ peroxide for 4-5 weeks; decalcified for 4 weeks in Sihler's I solution (1 portion glacial acetic acid, 2 portion of glycerin, and 12 portion $1 \%$ chloral hydrate); stained in Sihler's II solution (1 Ehrilch dyeing liquid, 2 copies glycerin, and 12 copies of $1 \%$ chloral hydrated) for 4 weeks; decolorized in Sihler's I solution for 2-10 hours; neutralized in $0.05 \%$ lithium carbonate solution for 2 hours; and soaked in steps in gradient glycerine $(40 \%, 60 \%, 80 \%$, $100 \%$ ) for 1 week to obtain transparency. On an X-ray reading box, the distribution of the intramuscular nerve branches were carefully observed. The percentage position of INDR and CINDR on the muscle length (from the origin to the insertion) and muscle width (from the upper edge to the lower edge) were measured using a Vernier caliper followed with photography and drawing. The drawn pattern of intramuscular nerve distribution was then placed back to the opposite side of the appropriate muscle of the same cadaver where the rotator cuff muscles were still intact. The drawing functioned to localize CINDRs of the muscle in reference to body surface landmarks (described below).

Reference line design: In order to locate the positions of CINDRs on body surface, the connecting line between the superior angle of scapula (point a) and the inferior angle of scapula (point b) is defined as the longitudinal (L) reference line, and the connecting line between the superior angle of scapula and the acromion (point c) as the horizontal $(\mathrm{H})$ reference line.

Localization of CINDR By Spiral computed tomography: According to the results of Sihler's staining, the corresponding position of CINDR was found on the contralateral rotator cuff muscles, and then labeled with barium sulfate, The CINDR was localized according to the method of spiral computed tomography (Siemens, Germany) (Fig. 1) which we used before to localize the nerve entry point (Yang et al., 2016; Chen et al., 2018). For the supraspinatus, infraspinatus and teres minor muscles, the projection point of CINDR on body surface of back was named as point $\mathrm{P}$, the intersection of the longitudinal line through point $\mathrm{P}$ and the line $\mathrm{H}$ and the horizontal line through 

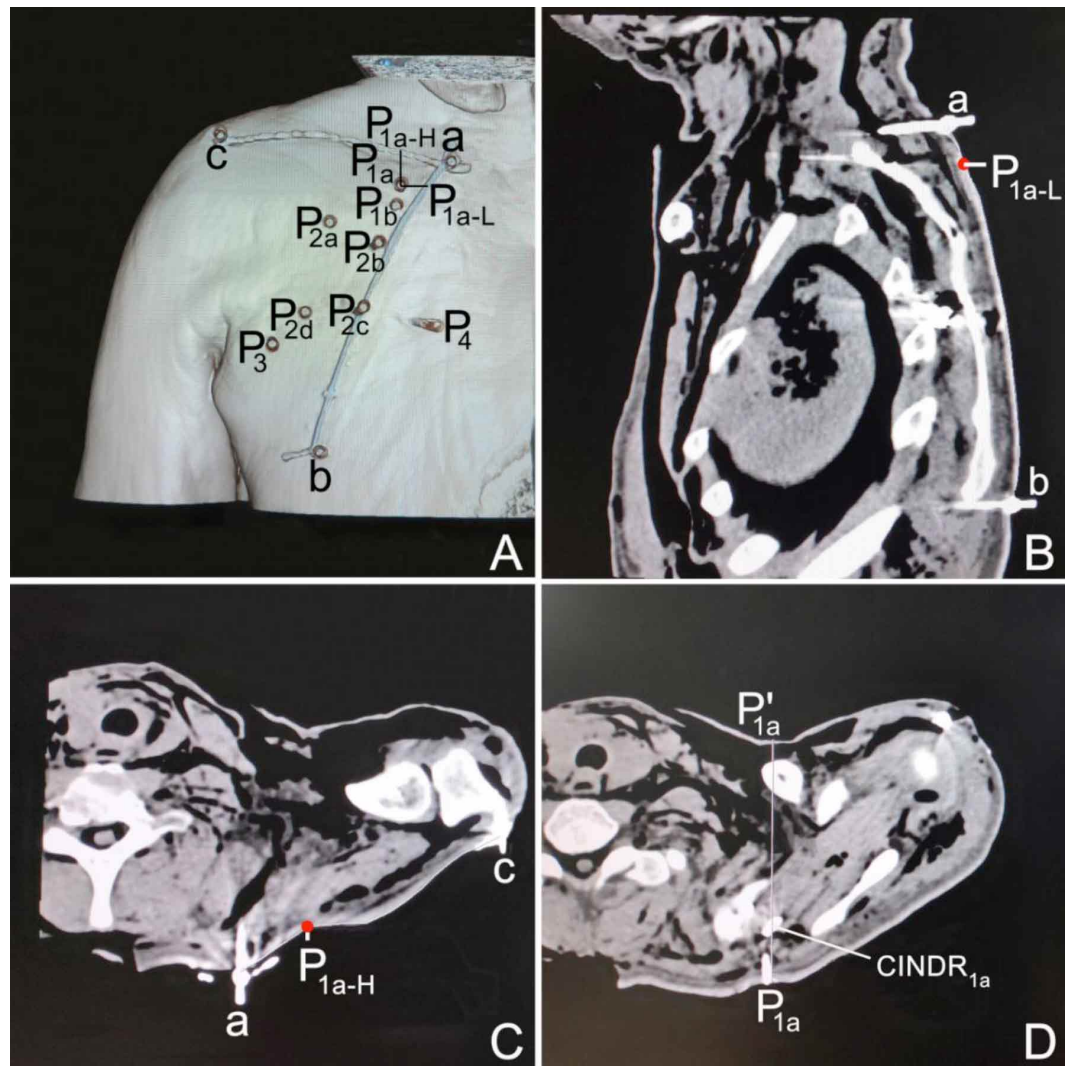

Fig. 1. CT image of the center of intramuscular nerve dense region of the left rotator cuff muscles (CINDR ${ }_{1 \mathrm{a}}$ as an example). A: Spiral CT three-dimensional reconstruction image shows the projection positions of the CINDR on the body surface and the designed reference lines. B: Lengths of lines $\mathrm{L}$ and a- $\mathrm{P}_{1 \mathrm{a}-\mathrm{L}}$ measured on the sagittal section. C: Lengths of lines $\mathrm{H}$ and $\mathrm{a}-\mathrm{P}_{1 \mathrm{a}-\mathrm{H}}$ measured on the transverse section. D: Depth of $\mathrm{CINDR}_{1 \mathrm{a}}$ measured on the transverse section.

point $\mathrm{P}$ and the line $\mathrm{L}$ were recorded as $\mathrm{P}_{\mathrm{H}}$ and $\mathrm{P}_{\mathrm{L}}$, respectively. The projection of CINDR on anterior thoracic wall across the transverse plane was named point $\mathrm{P}^{\prime}$. Percentage positions of $\mathrm{P}_{\mathrm{H}}$ and $\mathrm{P}_{\mathrm{L}}$ on lines $\mathrm{H}$ and $\mathrm{L}$ and the depths of CINDR were determined under the Syngo system (Siemens, Germany). For the subscapular muscle, the intersection point of the horizontal line at CINDR level and the medial edge of the scapula on body surface of back is named point $\mathrm{P}$, and the intersection point with the lateral edge of the scapula is named point $\mathrm{P}^{\prime}$. The percentile puncture depth was calculated as P-CINDR/P-P'.

After measurement and CINDR localization, these rotator cuff muscles were removed and subjected to Sihler's staining to verify whether intramuscular nerve distribution patterns and CINDR locations were consistent with those of the contralateral side muscles.

Statistical analysis. SPSS17.0 (SPSS Inc., Chicago, IL, USA) software was used for data analysis. The experimental data were expressed as percentage ( $\mathrm{n} \%$ ) to eliminate the influence of individual differences. Paired t test was used to compare the data between the right and left muscles. The comparison between men and women was performed using a two-sample t-test, test level was $\mathrm{a}=0.05$.

\section{RESULTS}

Sihler's staining revealed patterns of intramuscular nerve distribution Supraspinatus: The nerve for supraspinatus muscle originates from the suprascapular nerve and enters the muscle from the central part of the muscle belly near the upper margin deep in the muscle. It passes down to the central part of the muscle and divides into two primary branches: the superior branch and the inferior branch. The superior branch is in horizontal position and travels to the inner side (the origin) sending out secondary, tertiary, and terminal branches to the upper and lower regions along the way, especially more arborized branches at the end, forming INDR $_{1 \mathrm{a}}$. The inferior branch runs inwards, and sends out arborized branches at its end forming INDR $_{1 b}$ below $\operatorname{INDR}_{1 \mathrm{a}}$ (Fig. 2).

Infraspinatus: After the suprascapular nerve travels through the spinoglenoid notch to the deep surface of the infraspinatus, it enters the muscle at the upper margin of middle to outer $1 / 3$ of the muscle belly. Then, it divides into 3 primary branches going superior, medial, and inferior respectively. The superior branch is further divided into two secondary branches. One of the secondary branches sends out dense tree-like branches to the upper region, forming $\operatorname{INDR}_{2 \mathrm{a}}$, while the other secondary branch also sends out dense tree-like branches to the upper and terminal regions, forming INDR Ib $_{\text {. These }}$ two INDRs are near the upper middle margin of the muscle. The medial branch moves towards the origin of the muscle and gives off a arborized branch forming $\mathrm{INDR}_{2 \mathrm{c}}$ in the medial $1 / 3$ of the muscle. The inferior branch is parallel to the lower margin and runs obliquely inwards, with dense lower branches on the medial 1/3 of the muscle, forming INDR $_{2 \mathrm{~d}}$. There's an overlap region between INDR $_{2 c}$ and $\mathrm{INDR}_{2 \mathrm{~d}}$ (Fig. 3). 

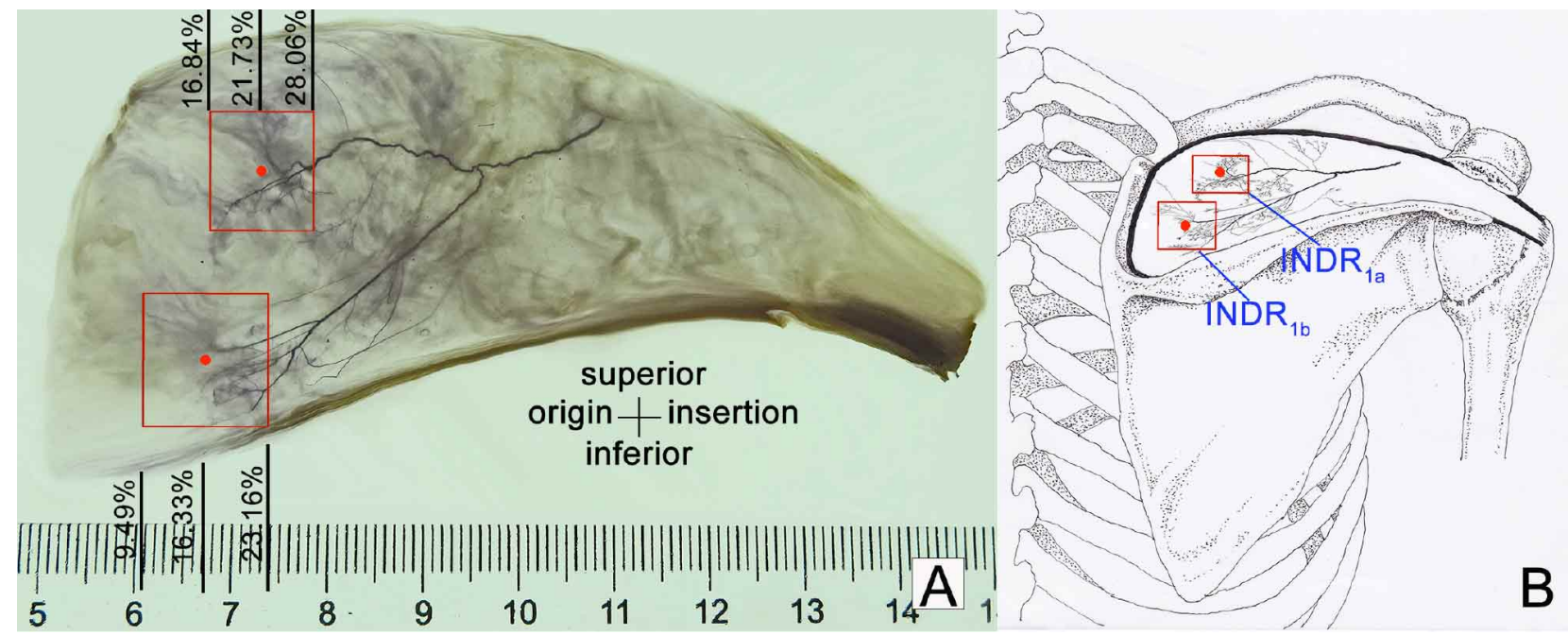

Fig. 2. Intramuscular nerve distribution pattern in the right supraspinatus and location of CINDR. A: Sihler's Staining demonstrates intramuscular nerve distribution patterns. Scale for cm. B: Schematic drawing of (A) and the positions of CINDRs. Red boxes and dots respectively INDR and CINDR.
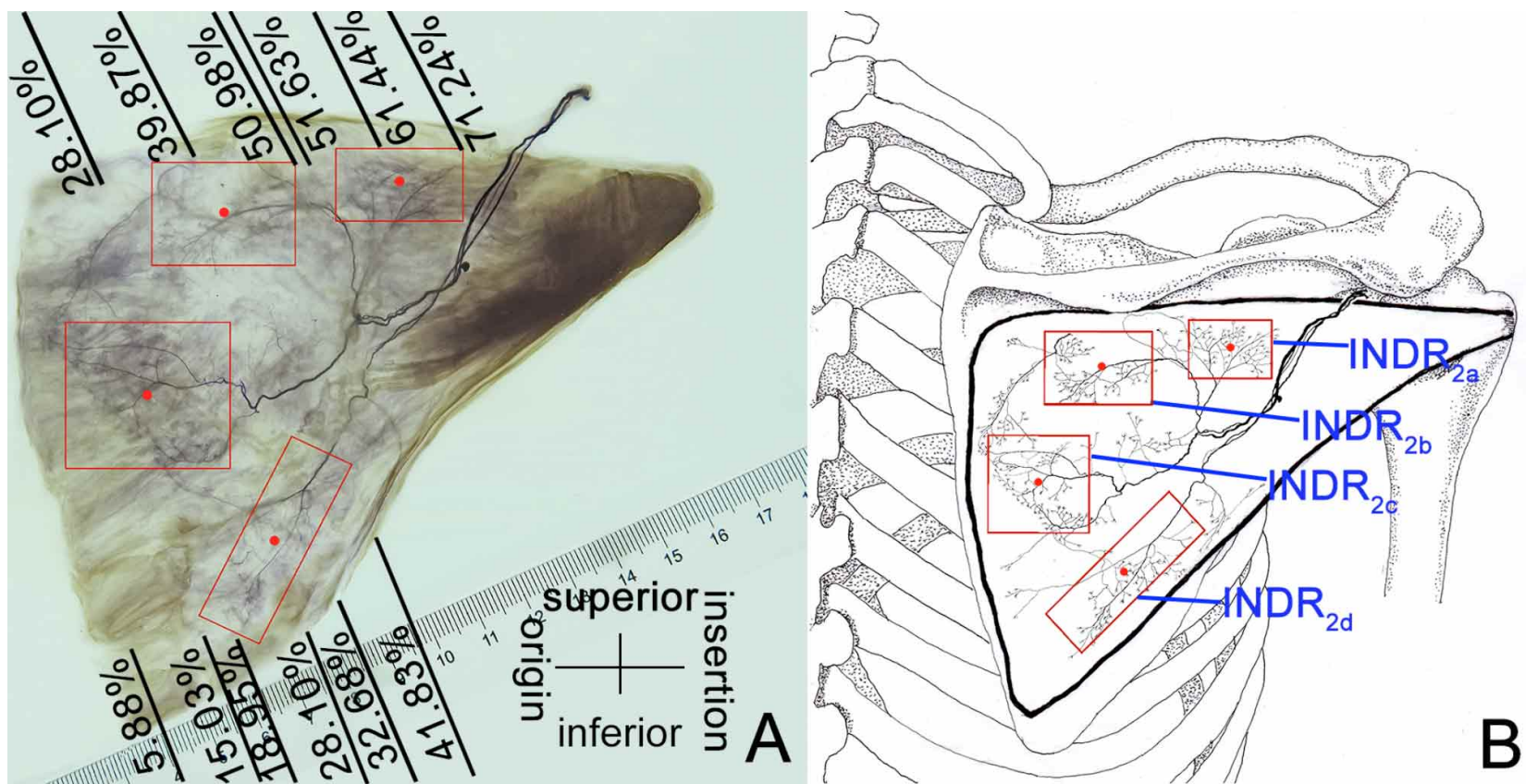

Fig. 3. Intramuscular nerve distribution pattern in the right infraspinatus and location of CINDR. A: Sihler's Staining demonstrates intramuscular nerve distribution patterns. Scale for $\mathrm{cm}$. B: Schematic drawing of (A) and the positions of CINDRs. Red boxes and dots respectively INDR and CINDR.

Teres minor: The nerve to teres minor muscle arise from the axillary nerve which exits the quadrangular space and then, enters the muscle from inferior at the medial and lateral $1 / 3$ junction of the muscle. After entering the muscle, it is divided into 3-4 primary branches, which go to the terminal, upper margin and the origin of the muscle respectively and send branches gradually. In particular, the branches that go to the upper margin and the origin are larger and have more branches to form an intramuscular nerve dense region, namely $\mathrm{INDR}_{3}$, which is located in the medial 1/3 of the muscle belly, as shown in Figure 4.

Subscapularis: The subscapularis is a multipennate muscle (often 7 penniform compartments) which is innervated by the upper and lower branches of the subscapular nerve. The upper nerve branch is thick and large. After the upper branch 


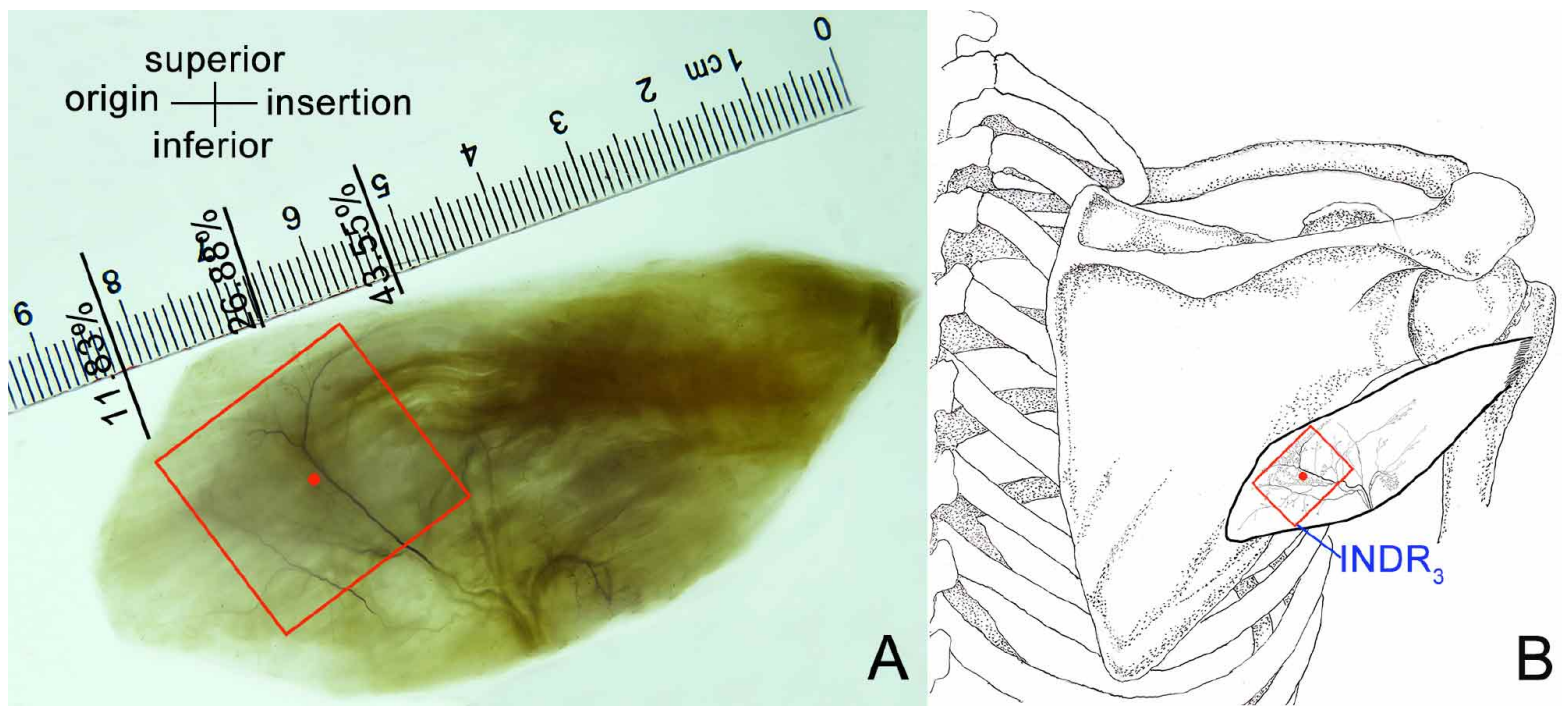

Fig.4. Intramuscular nerve distribution pattern in the right teres minor and location of CINDR . Scale for cm. A: Sihler's Staining demonstrates intramuscular nerve distribution patterns. Scale for $\mathrm{cm}$. B: Schematic drawing of (A) and the positions of CINDRs. Red boxes and dots respectively INDR and CINDR.

enters the muscle, it is first divided into two major primary branches. The superior primary branch is fan-shaped traveling to the origin (medial side) of the muscle and divided into 4 5 secondary branches. The inferior primary branch slopes downward and inwards, with 2 to 3 secondary branches. These secondary branches give off arborized branches and innervat the medial to the central part of the lower 3 compartments of the pinnate muscle. The lower branch of the subscapular nerve is relatively small and enters from the middle part of the lower margin of the muscle. It is divided into two branches and runs towards the medial side of the muscle. In the middle of the $6^{\text {th }}$ and $7^{\text {th }}$ compartments of the penniform muscle, the branches are overlapped with the branches of the upper branch, innervating the two compartments of the penniform muscle. These nerve branches often form a longitudinal intramuscular nerve dense regions in the medial center of the muscle (Fig. 5).
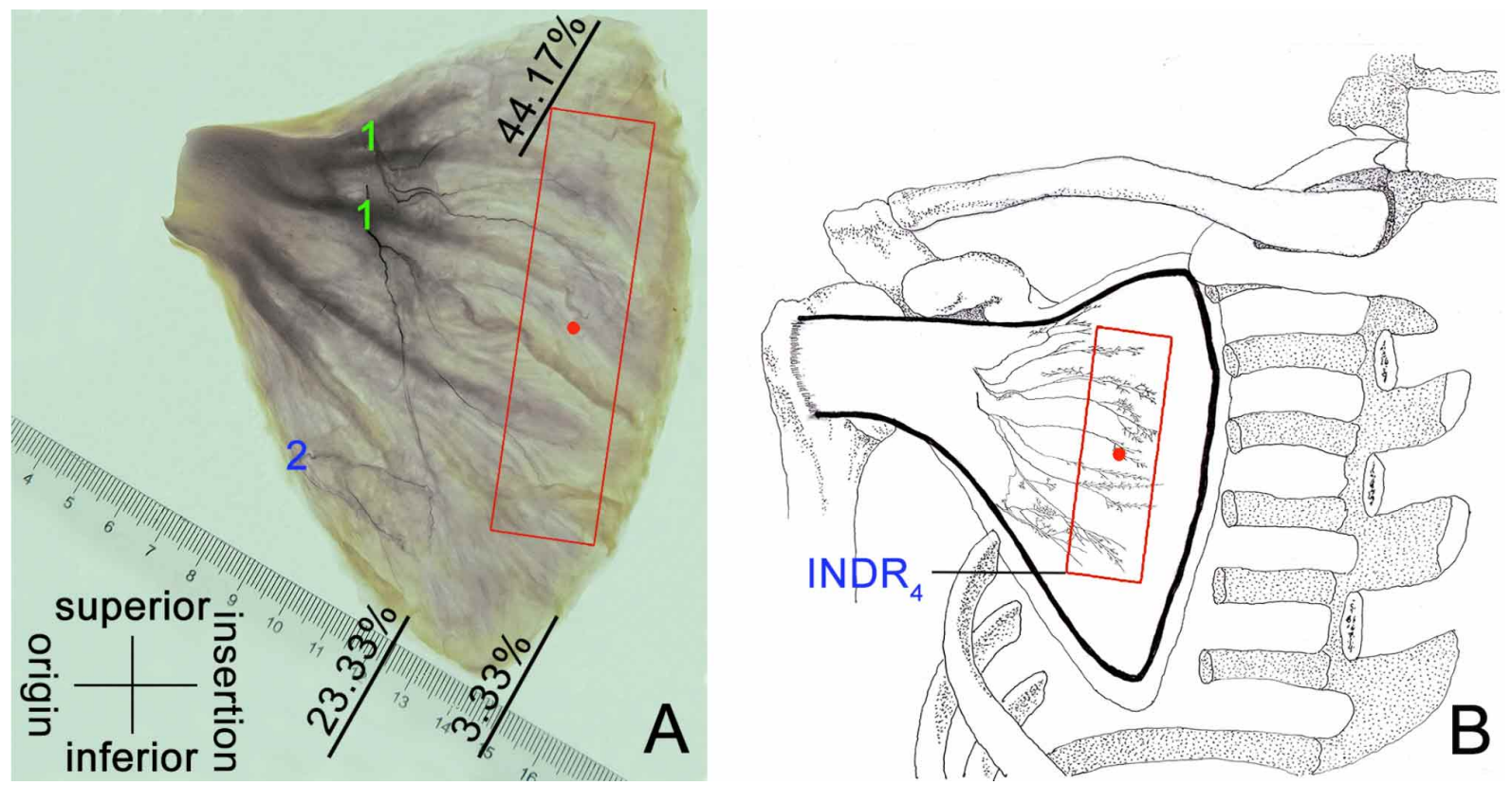

Fig. 5. Intramuscular nerve distribution pattern in the right subscapularis and location of CINDR. Scale for $\mathrm{cm}$. A: Sihler's Staining demonstrates intramuscular nerve distribution patterns. Scale for $\mathrm{cm}$. B: Schematic drawing of (A) and the positions of CINDRs. Red boxes and dots respectively INDR and CINDR .1= upper subscapular nerve, $2=$ lower subscapular nerve. 
Location of INDR and CINDR: The percentage position of center of INDRs on muscle length and width and area of INDRs are shown in Table I, and the location of CINDRs on Line $\mathrm{H}$ and Line $\mathrm{L}$ are shown in Table II. For women, the CINDRs in the infraspinatus and teres minor project below the level of the second rib on the chest, and are affected by the difference in breast size. So it is not appropriate to measure and calculate the percentage depth. Therefore, the absolute depth measured is $(2.42 \pm 0.03) \mathrm{cm},(1.85 \pm 0.03) \mathrm{cm}$, $(1.25 \pm 0.04) \mathrm{cm},(0.97 \pm 0.04) \mathrm{cm}$ and $(1.43 \pm 0.05) \mathrm{cm}$. There was no significant difference between left and right sides and between male and female, $\mathrm{P}>0.05$.

Table I. The percentage position of CINDRs on muscle length and width and area of INDRs.

\begin{tabular}{lccc}
\hline INDRs & $\begin{array}{c}\text { Location on muscle } \\
\text { length }(\%)\end{array}$ & $\begin{array}{c}\text { Location on muscle } \\
\text { width }(\%)\end{array}$ & Area_cm \\
\hline $\mathrm{INDR}_{1 \mathrm{a}}$ & $21.73 \pm 0.85$ & $30.61 \pm 1.08$ & $1.35 \pm 0.02$ \\
$\mathrm{INDR}_{\text {h }}$ & $16.33 \pm 0.84$ & $69.39 \pm 1.21$ & $1.92 \pm 0.03$ \\
$\mathrm{INDR}_{\text {, }}$ & $61.44 \pm 1.64$ & $13.28 \pm 0.63$ & $3.67 \pm 0.03$ \\
$\mathrm{INDR}_{2 \mathrm{~b}}$ & $39.87 \pm 1.56$ & $17.19 \pm 0.57$ & $6.45 \pm 0.04$ \\
$\mathrm{INDR}_{\text {, }}$ & $18.95 \pm 1.02$ & $46.88 \pm 1.29$ & $10.35 \pm 0.09$ \\
$\mathrm{INDR}_{{ }_{\text {a }}}$ & $28.10 \pm 1.01$ & $69.14 \pm 1.71$ & $5.75 \pm 0.05$ \\
$\mathrm{INDR}_{3}$ & $26.88 \pm 1.25$ & $39.17 \pm 1.37$ & $5.51 \pm 0.04$ \\
$\mathrm{INDR}_{4}$ & $23.33 \pm 1.18$ & $47.16 \pm 1.22$ & $19.36 \pm 0.10$ \\
\hline
\end{tabular}

Table II. The location of the $\mathrm{P}_{\mathrm{H}}$ and $\mathrm{P}_{\mathrm{L}}$ of CINDRs on Lines $\mathrm{H}$ and $\mathrm{L}$ respectively and the depth of CINDRs ( \%).

\begin{tabular}{cccc}
\hline CINDRs & $\mathrm{a}-\mathrm{P}_{\mathrm{H}} / \mathrm{H}$ & $\mathrm{a}-\mathrm{P}_{\mathrm{L}} / \mathrm{L}$ & $\mathrm{P}-\mathrm{CINDR} / \mathrm{PP}$ \\
\hline $\mathrm{CINDR}_{1 a}$ & $25.43 \pm 0.61$ & $11.09 \pm 0.07$ & $24.83 \pm 0.27$ \\
CINDR $_{1 \mathrm{~b}}$ & $26.59 \pm 0.83$ & $14.83 \pm 0.56$ & $25.40 \pm 0.37$ \\
CINDR $_{\text {a }}$ & $53.85 \pm 1.92$ & $21.59 \pm 0.72$ & $21.55 \pm 0.55$ \\
CINDR $_{\text {, }}$ & $34.63 \pm 1.08$ & $27.93 \pm 0.82$ & $16.10 \pm 0.14$ \\
CINDR $_{2 \mathrm{c}}$ & $35.96 \pm 0.52$ & $48.55 \pm 1.41$ & $10.01 \pm 0.16$ \\
$\mathrm{CINDR}_{\text {, }}$ & $58.17 \pm 0.91$ & $57.52 \pm 1.39$ & $8.14 \pm 0.53$ \\
$\mathrm{CINDR}_{2}$ & $74.50 \pm 0.69$ & $68.28 \pm 1.02$ & $13.27 \pm 0.73$ \\
$\mathrm{CINDR}_{4}$ & $20.33 \pm 0.55$ & $52.82 \pm 1.18$ & $22.88 \pm 0.55$ \\
\hline
\end{tabular}

\section{DISCUSSION}

The four rotator cuff muscles cover the upper, posterior and anterior of the glenohumeral joint respectively, and merge with the joint capsule to maintain joint stability in the state of motion or rest (Sgroi \& Cilenti, 2018). In some central nervous system diseases, when spasticity occur in the supraspinatus, infraspinatus and teres minor, the shoulder joints show continuous abduction and rotation. When subscapularis spasticity occurs, the shoulder joint presents continuous adduction, pronation and mild extension (Chironna et al., 1990; de Boer et al., 2008). These continuous muscle spasms may lead to limited shoulder joint movement with shoulder pain, which seriously affect the daily life of patients. Therefore, to relieve the spasticity of these muscles and restore the affected limb's free movement is an important task in current rehabilitation medicine.

Currently, chemical nerve block by intramuscular injection of BTX-A is a commonly used therapeutic method to relieve muscle spasticity (Yelnik et al., 2003; Pedreira et $a l$.). However, for BTX-A to achieve its maximum efficacy, the injection location should be as close as possible to its effector site, which is the motor endplate (Childers et al., 2004). If the injection site of BTX-A deviates from the moving endplate by $5 \mathrm{~mm}$, the effect will be reduced by $50 \%$ (Parratte et al., 2002). Therefore, the accurate localization of BTX-A blocking target is the key to obtain a curative effect. However, it is not realistic to localize the motor endplate through staining. Since the motor endplate position is consistent with INDR and the INDR of many muscles has been revealed (Yang et al.; Tang et $a l$.), it is possible to utilize INDR as the target for BTX-A injection. The microscopic study of rotator cuff muscles by Warden et al. showed that $78 \%$ of the nerves innervating the subscapularis muscle were divided into three branches with two upper and one lower innervating the muscle belly of the upper, middle and lower parts of the muscle. Only $22 \%$ of this nerves branches into two branches as superior and inferior branches of the 
subscapular nerve (Warden et al.). Tae-hyeon Cho et al. used Sihler's staining technique to stain subscapularis, and found that $100 \%$ of subscapularis had the upper branch of subscapular nerve, and only $80 \%$ of subscapularis had the lower branch of the nerve. In this study, it was found that all muscle masses had lower branches of the subscapular nerve, possibly due to the number of specimens. However, both studies recommended BTX-A injections in the middle of the muscle. From the results of this study, it can be seen that the middle part of this muscle is not INDR, and the INDR is near the middle part of the medial muscle. Therefore, we recommend to inject BTX-A on back at $50.14 \%$ on the line $\mathrm{L}$ and $22.88 \%$ on line $\mathrm{H}$ at the inside of edge of the scapula.

In fact, the nerve block approach of rotator cuff spasticity with shoulder pain has been reported in the upper, lateral and medial scapula (Chironna et al.; Rha et al.). In this study, the location of intramuscular nerve dense regions in the 4 rotator cuff muscles was comprehensively considered, and the connection line between the superior angle of scapula and the acromion was designed as a transverse reference line to describe the inside and outside relationship between CINDRs and bone markers. The superior angle of scapula and the inferior angle of scapula are the longitudinal reference lines, which are used to describe the upper and lower relationship between CINDRs and bone markers. This allows the insertion of all four muscles into the back. The data are expressed as percentages, which can exclude the influence of individual differences.

Increasing the dose of BTX-A within a certain range can improve the treatment effect, but long-term high-dose use may lead to partial muscle fibrosis and excessive muscle relaxation, resulting in poor long-term therapy result (Ghasemi et al., 2013). Studies have shown, 1U BTX-A can infiltrate about 1.50-3.00 $\mathrm{cm}^{2}$, and 2.5-10U can infiltrate about 4.50 $\mathrm{cm}^{2}$ (Borodic et al., 1994). According to the results, there were two INDRs in the supraspinatus, of which the area of INDR was $1.35 \mathrm{~cm}^{2}$, and $1 \mathrm{U}$ of BTX-A should be injected. The area of INDR ${ }_{1 b}$ was $1.91 \mathrm{~cm}^{2}$, and only $1 \mathrm{U}$ was needed. There are 4 INDRs in the infraspinatus, and a total of $20 \mathrm{U}$ is needed for the same calculation. However, Lim et al. reported that at least two sites should be injected into this muscle, and the injection dose at each point should not exceed $25 \mathrm{U}$, and the dose of the whole muscle should not exceed $50 \mathrm{U}$ (Lim et al., 2008). The dose of $20 \mathrm{U}$ in this study is much smaller than the recommended above. According to the results of this study, it can be concluded that $4.5 \mathrm{U}$ of BTX-A should be injected into the teres minor to obtain a better therapeutic effect.

For subscapularis, Unlu et al. injected BTX-A in the thickest part of subscapularis. In this study, it was found that the thickest part of the muscle was the outer and lower part of the muscle, and only the lower branches of the subscapular nerve were distributed in this part, which was not a densely nerve area. The INDR area of this muscle is 19.36 $\mathrm{cm}^{2}$. According to the above calculation principle, BTX-A required by this muscle is about $16 \mathrm{U}$.

Overall, this study, through improved Sihler's staining method, showed the INDR of rotator cuff muscles and used body surface boney landmarks as references to locate the CINDRs. CT scan was used to establish the geometric relationship between the body surface puncture location and puncture depth of the CINDRs. We also evaluated the required dose usage of BTX-A. These results may provide an accurate target for BTX-A injection in the treatment of rotator cuff muscles spasticity with shoulder pain, and may help to improve the efficiency and efficacy of targeted nerve blocks. However, its efficacy remains to be verified clinically.

\section{CONCLUSIONS}

The location of these CINDRs provides the optimal target location for the injection of botulinum toxin $\mathrm{A}$ in treatment of rotator cuff muscles spasticity with shoulder pain. This may provide help to improve the efficiency and efficacy of targeted nerve blocks.

\section{ACKNOWLEDGMENTS}

The authors would like to acknowledge Yanrong Li for her excellent technical assistance and use of their spiral computed tomography laboratory. We also would like to express our sincere gratitude to the 20 body donors who were anonymous teachers helped us to complete the project. This work was supported by the National Natural Science Foundation of China (31540031, 31660294) and the Master's fund project of Zunyi Medical University (F-761).

LUO, H.; JI, S. \& YANG, S. Localización de los centros de las regiones densas del nervio intramuscular de los músculos del manguito rotador: una guía para la inyección de toxina botulínica A por espasticidad con dolor de hombro. Int. J. Morphol., 38(2):435443, 2020.

RESUMEN: Con el objetivo de localizar con precisión los centros de las regiones densas del nervio intramuscular (CRDNI) de los músculos del manguito rotador, se utilizaron veinte cadáveres adultos. Las curvas en la piel que conectan el ángulo superior de la escápula con el acromion y con el ángulo inferior de la escápula se determinaron como líneas de referencia horizontales $(\mathrm{H})$ 
y longitudinales (L), respectivamente. Se extrajo de un lado los músculos del manguito rotador y se sometió a la tinción de Sihler para mostrar regiones densas de nervios intramusculares, y los CRDNI de los músculos contralaterales se marcaron con sulfato de bario y se escanearon mediante tomografía computarizada (para determinar los puntos de proyección de la superficie corporal (P)). La intersección de la línea longitudinal desde el punto $\mathrm{P}$ a la línea $\mathrm{H}$, y de la línea horizontal desde el punto $\mathrm{P}$ a la línea $\mathrm{L}$, se registraron como $\mathrm{PH}$ y PL, respectivamente. La proyección de CRDNI en la superficie del cuerpo anterior a través del plano sagital se definió como $\mathrm{P}$ 'y la línea que conecta $\mathrm{P}$ a $\mathrm{P}^{\prime}$ se registró como Línea $\mathrm{PP}$ '. Las posiciones porcentuales de los CRDNI de PH y PL en las líneas H y L, y las profundidades en la línea PP 'se determinaron bajo el sistema Syngo. Se identificaron dos, cuatro, uno y un CINDR en los músculos supraespinoso, infraespinoso, redondo menor y subescapular, respectivamente. Las posiciones de $\mathrm{PH}$ de estos CRDNI en la línea $\mathrm{H}$ son las siguientes: supraespinoso, $25,43 \%$ y $26.59 \%$; infraspinatus, $53,85 \%, 34,63 \%, 35,96 \%$ y $58,17 \%$; redondo menor, $74,50 \%$; y subescapular, 20,33\%. El PL en la línea L: supraespinoso, $11.09 \%$ y $14.83 \%$; infraspinatus, $21,59 \%, 27,93 \%, 48,55 \%$ y $57,52 \%$; redondo menor, $68.28 \%$; y subescapular, $52,82 \%$. La profundidad en la línea PP ': supraespinoso, $24,83 \%$ y $25,40 \%$; infraspinatus, 21,55 $\%, 16,10 \%, 10,01 \%$ y $8,14 \%$; redondo menor, $13.27 \%$; y subescapularis, 22,88 \%. La identificación de estos CRDNI debería proporcionar la posición objetivo óptima para inyectar la toxina botulínica A para tratar la espasticidad de los músculos del manguito rotador acompañada de dolor en el hombro y para mejorar la eficiencia y la eficacia del bloqueo de la localización del objetivo.

PALABRAS CLAVE: Músculos del manguito rotador; Espasticidad; Regiones densas del nervio intramuscular; Toxina botulínica A; Bloqueo de localización de destino.

\section{REFERENCES}

Amirali, A.; Mu, L.; Gracies, J. M. \& Simpson, D. M. Anatomical localization of motor endplate bands in the human biceps brachii. $J$. Clin. Neuromuscul. Dis., 9(2):306-12, 2007.

Borodic, G. E.; Ferrante, R.; Pearce, L. B. \& Smith, K. Histologic assessment of dose-related diffusion and muscle fiber response after therapeutic botulinum A toxin injections. Mov. Disord., 9(1):31-9, 1994.

Chae, J.; Mascarenhas, D.; Yu, D. T.; Kirsteins, A.; Elovic, E. P.; Flanagan, S. R.; Harvey, R. L.; Zorowitz, R. D. \& Fang, Z. P. Poststroke shoulder pain: its relationship to motor impairment, activity limitation, and quality of life. Arch. Phys. Med. Rehabil., 88(3):298-301, 2007.

Childers, M. K. Targeting the neuromuscular junction in skeletal muscles. Am. J. Phys. Med. Rehabil., 83(10 Suppl.):S38-44, 2004.

Childers, M. K.; Kornegay, J. N.; Aoki, R.; Otaviani, L. \& Petroski, G. Evaluating motor end-plate-targeted injections of botulinum toxin type A in a canine model. Muscle Nerve, 21(5):653-5, 1998.

Chironna, R. L. \& Hecht, J. S. Subscapularis motor point block for the painful hemiplegic shoulder. Arch. Phys. Med. Rehabil., 71(6):4289, 1990.

Cho, T. H.; Kwon, H. J.; Choi, Y. J.; Jehoon, O.; Won, S. Y. \& Yang, H. $M$. Intramuscular innervation of the subscapularis muscle and its clinical implication for the BoNT Injection: an anatomical study using the modified Sihler's staining. Clin. Anat., 32(1):110-6, 2019. de Boer, K. S.; Arwert, H. J.; de Groot, J. H.; Meskers, C. G. M.; Mishre, A. D. R. \& Arendzen, J. H. Shoulder pain and external rotation in spastic hemiplegia do not improve by injection of botulinum toxin A into the subscapular muscle. J. Neurol. Neurosurg. Psychiatry, 79(5):581-3, 2008.

Friedman, A.; Diamond, M.; Johnston, M. V. \& Daffner, C. Effects of botulinum toxin A on upper limb spasticity in children with cerebral palsy. Am. J. Phys. Med. Rehabil., 79(1):53-9, 2000.

Ghasemi, M.; Salari, M.; Khorvash, F. \& Shaygannejad, V. A Literature review on the efficacy and safety of botulinum toxin: an injection in post-stroke spasticity. Int. J. Prev. Med., 4(Suppl. 2):S147-58, 2013.

Hecht, J. S. Subscapular nerve block in the painful hemiplegic shoulder. Arch. Phys. Med. Rehabil., 73(11):1036-9, 1992.

Joynt, R. L. The source of shoulder pain in hemiplegia. Arch. Phys. Med. Rehabil., 73(5):409-13, 1992.

Kinnett, D. Botulinum toxin A injections in children: technique and dosing issues. Am. J. Phys. Med. Rehabil., 83(10 Suppl.):S59-64, 2004.

Lim, J. Y.; Koh, J. H. \& Paik, N. J. Intramuscular botulinum toxin-A reduces hemiplegic shoulder pain: a randomized, double-blind, comparative study versus intraarticular triamcinolone acetonide. Stroke, 39(1):126-31, 2008.

Maynard, F. M.; Karunas, R. S. \& Waring 3rd, W. P. Epidemiology of spasticity following traumatic spinal cord injury. Arch. Phys. Med. Rehabil., 71(8):566-9, 1990.

Park, J. \& Chung, M. E. Botulinum toxin for central neuropathic pain. Toxins (Basel), 10(6):224, 2018.

Parratte, B.; Tatu, L.; Vuillier, F.; Diop, M. \& Monnier, G. Intramuscular distribution of nerves in the human triceps surae muscle: anatomical bases for treatment of spastic drop foot with botulinum toxin. Surg. Radiol. Anat., 24(2):91-6, 2002.

Pedreira, G.; Cardoso, E. \& Melo, A. Botulinum toxin type A for refractory post-stroke shoulder pain. Arq. Neuropsiquiatr., 66(2A):213-5, 2008.

Picelli, A.; Bonazza, S.; Lobba, D.; Parolini, M.; Martini, A.; Chemello, E.; Gandolfi, M.; Polati, E.; Smania, N. \& Schweiger, V. Suprascapular nerve block for the treatment of hemiplegic shoulder pain in patients with long-term chronic stroke: a pilot study. Neurol. Sci., 38(9):1697701, 2017.

Rasool, G.; Afsharipour, B.; Suresh, N. L. \& Rymer, W. Z. Spatial analysis of multichannel surface EMG in hemiplegic stroke. I. E. E. E. Trans. Neural Syst. Rehabil. Eng., 25(10):1802-11, 2017.

Renzenbrink, G. J. \& Ijzerman, M. J. Percutaneous Neuromuscular Electrical Stimulation (P-NMES) for treating shoulder pain in chronic hemiplegia. Effects on shoulder pain and quality of life. Clin. Rehabil., 18(4):359-65, 2004.

Rha, D. W.; Han, S. H.; Kim, H. J.; Won, S. Y. \& Lee, S. C. Ultrasoundguided lateral approach for needle insertion into the subscapularis for treatment of spasticity. Arch. Phys. Med. Rehabil., 93(7):1147-52, 2012.

Sgroi, T. A. \& Cilenti, M. Rotator cuff repair: post-operative rehabilitation concepts. Curr. Rev. Musculoskelet. Med., 11(1):86-91, 2018.

Shanahan, E. M.; Ahern, M.; Smith, M.; Wetherall, M.; Bresnihan, B. \& FitzGerald, O. Suprascapular nerve block (using bupivacaine and methylprednisolone acetate) in chronic shoulder pain. Ann. Rheum. Dis., 62(5):400-6, 2003.

Simpson, D. M.; Gracies, J. M.; Yablon, S. A.; Barbano, R.; Brashear, A. $\&$ BoNT/TZD Study Team. Botulinum neurotoxin versus tizanidine in upper limb spasticity: a placebo-controlled study. J. Neurol. Neurosurg. Psychiatry, 80(4):380-5, 2009.

Snels, I. A.; Beckerman, H.; Twisk, J. W.; Dekker, J. H.; De Koning, P.; Koppe, P. A.; Lankhorst, G. J. \& Bouter, L. M. Effect of triamcinolone acetonide injections on hemiplegic shoulder pain: a randomized clinical trial. Stroke, 31(10):2396-401, 2000.

Tang, S.; Zhang, M. X.; \& Yang, S. Localization of center of intramuscular nerve dense regions in adult anterior brachial muscles a guide for botulinum toxin A injection to treat muscle spasticity. Am. J. Transl. Res., 10(4):1220-8, 2018. 
LUO, H.; JI, S. \& YANG, S. Localization of the centers of intramuscular nerve dense regions of the rotator cuff muscles: A guide for botulinum toxin a injection for spasticity accompanied by shoulder pain. Int. J. Morphol., 38(2):435-443, 2020.

Unlu, E.; Sen, T.; Esmer, A. F.; Tuccar, E.; Elhan, A. \& Cakci, A. A new technique for subscapularis muscle needle insertion. Am. J. Phys. Med. Rehabil., 87(9):710-3, 2008.

Van Ouwenaller, C.; Laplace, P. M. \& Chantraine, A. Painful shoulder in hemiplegia. Arch. Phys. Med. Rehabil., 67(1):23-6, 1986.

Warden, J. M.; Roberts, S. L.; Chang, Y.; Baker, R.; Boulias, C.; Ismail, F. \& Agur, A. M. Neuromuscular partitioning of subscapularis based on intramuscular nerve distribution patterns: implications for botulinum toxin injections. Arch. Phys. Med. Rehabil., 95(7):140815, 2014.

Wassef, M. R. Suprascapular nerve block. A new approach for the management of frozen shoulder. Anaesthesia, 47(2):120-4, 1992.

Yang, F.; Zhang, X.; Xie, X.; Yang, S.; Xu, Y. \& Xie, P. Intramuscular nerve distribution patterns of anterior forearm muscles in children: a guide for botulinum toxin injection. Am. J. Transl. Res., 8(12):548593, 2016.

Yelnik, A. P.; Colle, F. M. C. \& Bonan, I. V. Treatment of pain and limited movement of the shoulder in hemiplegic patients with botulinum toxin A in the subscapular muscle. Eur. Neurol., 50(2):91-3, 2003.

Yelnik, A. P.; Colle, F. M.; Bonan, I. V. \& Vicaut, E. Treatment of shoulder pain in spastic hemiplegia by reducing spasticity of the subscapular muscle: a randomised, double blind, placebo controlled study of botulinum toxin A. J. Neurol. Neurosurg. Psychiatry, 78(8):845-8, 2007.

\author{
Corresponding author: \\ Shengbo Yang \\ Department of Anatomy \\ Zunyi Medical University \\ 6 University West Road \\ Xinpu New Developing Areas \\ Zunyi City \\ Guizhou Province \\ CHINA
}

Email: yangshengbo8205486@163.com

Received: 26-09-2019

Accepted: 28-10-2019 